\title{
REVISÃO SISTEMÁTICA E METANÁLISE: NÍVEIS DE EVIDÊNCIA E VALIDADE CIENTÍFICA
}

\author{
SYSTEMATIC REVIEW AND META-ANALYSIS: LEVELS OF EVIDENCE AND SCIENTIFIC VALIDITY
}

Hortência de Abreu Gonçalves, Marilene Batista da Cruz Nascimento, Kathia Cilene Santos Nascimento

Universidade Tiradentes

E-mail: ensino.pesquisa@yahoo.com.br, nascimentolene@yahoo.com.br, kathia.pesquisa@outlook.com

\section{Resumo}

A revisão sistemática constitui-se numa investigação literária que possibilita a síntese criteriosa das evidências disponíveis sobre um problema específico, servindo para auxiliar ao desenvolvimento da informação científica, associada ou não à metanálise. Objetivou-se apresentar os fundamentos teóricos da revisão sistemática e metanálise, ressaltando a importância dos níveis de evidência e validade ao estudo quantitativo e ao qualitativo. Recorreu-se ao levantamento de fontes bibliográficas digitais sobre essas temáticas em bases de dados científicas e universitárias. Buscou-se ainda, o método de análise de conteúdo como eixo norteador do estudo, numa perspectiva explicativa, pautada na síntese dos argumentos verificados nas fontes investigadas.

Palavras-chave: revisão sistemática. literatura. metanálise.

\section{Abstract}

The systematic review is it a literary research that enables the careful synthesis of available evidence about a particular problem, serving to assist the development of scientific information, with or without meta-analysis. This review article presents the theoretical foundations of systematic review and meta-analysis, highlighting the importance of levels of evidence and validity study the quantitative and the qualitative. He appealed to the survey of digital library resources on these topics in scientific and university databases. It attempted still, the method of analysis of content as a guideline of the study, an explanatory perspective, based on the arguments checked in the investigated sources.

Palavras-chave: systematic review. literature. meta-analysis. 


\section{INTRODUÇÃO}

A revisão sistemática pode ser definida como "uma forma de pesquisa que utiliza como fonte de dados a literatura sobre determinado tema" (SAMPAIO; MANCINI, 2007, p.83), possibilitando uma investigação que visa identificar evidências relacionadas a um problema específico de pesquisa, com o intuito de destacar ideias, posturas e opiniões de autores, publicadas na área de conhecimento em que se insere. Tendo como principal meta, "caracterizar cada estudo selecionado, avaliar a qualidade deles, identificar conceitos importantes, comparar as análises estatísticas apresentadas e concluir sobre o que a literatura informa em relação a determinada intervenção, apontando ainda problemas/questões que necessitam de novos estudos" (SAMPAIO; MANCINI, 2007, p. 84). Ressalta-se ainda que esse tipo de investigação possibilita a elaboração do "resumo das evidências relacionadas a uma estratégia de intervenção específica, mediante a aplicação de métodos explícitos e sistematizados de busca, apreciação crítica e síntese da informação selecionada" (SAMPAIO; MANCINI, 2007, p.64), fundamentando propostas de mudanças nas áreas de prevenção, diagnóstico, tratamento e reabilitação por meio da elaboração de uma base integrativa referendada nos estudos de metanálise, indispensáveis à prática médica baseada em evidências que visam a sustentabilidade científica.

Diante disso, surgem indagações pertinentes, tais como: Em que consiste a evidência e a validade científica? Por que a revisão sistemática e a metanálise são prioritárias aos estudos no contexto da investigação em saúde? A sustentação teórica da revisão sistemática visa também ao estado da arte? Em que medida é utilizada a abordagem qualitativa e quantitativa em estudos de revisão sistemática? Em que consiste a metodologia empregada na revisão sistemática e de que forma é planejada? Quais as vantagens e as desvantagens dos estudos de revisão sistemática? Este artigo objetiva apresentar os fundamentos teóricos da revisão sistemática e metanálise, ressaltando a importância dos níveis de evidência e validade ao estudo quantitativo e ao qualitativo na pesquisa científica. Recorreu-se ao levantamento de fontes bibliográficas digitais sobre esses conteúdos em repositórios científicos e universitários. Para o entendimento e a verificação das questões abordadas, 
buscou-se, sustentação teórica no método de análise de conteúdo, eixo norteador do estudo, numa perspectiva explicativa, pautada na síntese dos argumentos evidenciados na literatura pesquisada.

\section{REVISÃO SISTEMÁTICA}

O levantamento dos estudos publicados para a realização da revisão sistemática necessita da observação de algumas etapas, sendo elas: definição do problema de pesquisa, elaboração de protocolo de estudos, determinação dos termos técnicos e palavras-chave da pesquisa para a identificação das evidências, estabelecimento de critérios de inclusão e exclusão para as publicações, análise crítica e avaliativa do referencial teórico selecionado e resumo das informações obtidas e ainda, as conclusões alcançadas, conforme descrito no quadro 1.

Quadro 1. Etapas de uma revisão sistemática.

\begin{tabular}{|ll|}
\hline 1. & Problema de Pesquisa (questão a ser investigada) \\
2. & Protocolo de Pesquisa (descrição criteriosa do estudo) \\
4. & Bases de Dados (localização dos estudos) \\
5. & Análise, Crítica e Avaliação (validade dos estudos selecionados) \\
6. & Elaboração do Resumo (Síntese dos conteúdos abordados) \\
7. & Identificação das Evidências (estudos agrupados conforme a semelhança) \\
8. & Conclusão (alcance das evidências identificadas) \\
\hline
\end{tabular}

Fonte: dados da pesquisa (2015).

De acordo com Munzlinger, Narcizo e Queiroz (2012, não paginado), a revisão sistemática compreende um processo de pesquisa referendado em estudos bibliográficos, com o intuito de proceder ao levantamento formal

do estado da arte, de forma robusta e consistente, a partir de um planejamento e execução criteriosos [Biolchini et al., 2005; Pai et al., 2004; Cochrane, 2003; Kan et al., 2001]. As [...] [revisões sistemáticas] são planejadas para serem metódicas, explícitas e passíveis de reprodução 
[Sampaio e Mancini, 2007]. Para tanto, o processo de pesquisa é conduzido segundo uma sequência metodologicamente bem definida de etapas, de acordo com um protocolo de estudo previamente planejado.

A elaboração de um protocolo de revisão sistemática deve contemplar os seguintes componentes: "a pergunta da revisão, os critérios de inclusão, as estratégias para buscar as pesquisas, como as pesquisas serão avaliadas criticamente, a coleta e a síntese dos dados" (GALVÃO; SAWADA; TREVISAN, 2004, p. 551), com a finalidade de utilização durante a realização da revisão sistemática para evidenciar, congregar e avaliar criticamente os estudos relevantes, considerando nessa busca a abrangência temporal das fontes secundárias e o estabelecimento do tamanho da amostra que será objeto de estudo. E ainda, "formalizar o estudo primário e definir, acompanhar e documentar todos os passos a serem executados no estudo secundário de Revisão Sistemática" (MUNZLINGER; NARCIZO; QUEIROZ, [s.d.], não paginado).

Em seu planejamento, o protocolo deve detalhar as etapas metodológicas do estudo que será realizado, visando à promoção dos métodos e a conduta científica adotada, possibilitando, assim, a revisão pelos pares, tanto para a abordagem qualitativa, quanto para a quantitativa. Entretanto, sendo comum a essas abordagens, a presença de alguns aspectos metodológicos, dentre eles: "justificativa, [...] objetivos, critérios de inclusão, tipo de estudo para inclusão, estratégia de busca, critérios para avaliar a validade dos estudos, formas de extração e síntese das [...] [evidências], formas de apresentação dos resultados e declaração de conflito de interesses" (DE-LA-TORRE-UGARTE-GUANILO; TAKAHASHI; BERTOLOZZI, 2011, p.1265). O termo evidência tem sido usado com frequência em estudos da área da saúde, especialmente relacionado à tomada de decisões (GALVÃO; SAWADA; TREVISAN, 2004, p. 551).

Quando finalizado, o "protocolo deve ser submetido ao Cochrane Review Group ou Evidence Synthesis Groups (JBI), que pode solicitar modificações para aprimorar a metodologia. Quando aceito, o protocolo será publicado em suas respectivas bases de dados" (DE-LA- 
TORRE-UGARTE-GUANILO; TAKAHASHI; BERTOLOZZI, 2011, p.1265). Cabe destacar que a "Colaboração Cochrane [...] é responsável pela elaboração e disseminação de revisões sistemáticas que retratam a eficácia de intervenções na área da saúde" (MENDES; SILVEIRA; GALVÃO, 2008, não paginado). Complementando, essa fundação objetiva "preparar, manter e disseminar revisões sistemáticas na área da Saúde" (CASTRO, 2007, não paginado). Com esse intuito, instalou na Europa "sete centros Cochrane (França, Alemanha, Grã-Bretanha, Espanha, Itália, Holanda e Dinamarca), além de centros no Canadá, China, Austrália, Nova Zelândia, África do Sul e Brasil" (CASTRO, 2007, não paginado).

\subsection{Contraponto entre revisão sistemática e revisão tradicional ou narrativa}

Adentrando ao cerne das revisões sistemáticas e suas metodologias de atuação, cabe enfatizar que:

os "sujeitos" da investigação são os estudos primários (unidades de análise) selecionados por meio de método sistemático e pré-definido. Os estudos primários podem ser ensaios clínicos aleatórios, estudos de acurácia, estudos de coortes ou qualquer outro tipo de estudo. A escolha do tipo de estudo depende da pergunta que se pretende responder. Tradicionalmente, a revisão sistemática é um estudo retrospectivo. Existe ainda a possibilidade de realizar a revisão sistemática com dados individuais (CASTRO, 2007, não paginado).

Nessa perspectiva, pode-se afirmar que a revisão sistemática difere da revisão narrativa ou tradicional em alguns critérios, assim especificados por Rother (2007, não paginado), no quadro 2: 
Quadro 2. Diferenças entre revisão sistemática e revisão narrativa.

\begin{tabular}{|l|l|l|}
\hline \multicolumn{1}{|c|}{ Itens } & \multicolumn{1}{|c|}{ Revisão Narrativa } & \multicolumn{1}{c|}{ Revisão sistemática } \\
\hline Questão & Ampla & Específica \\
\hline Fonte & $\begin{array}{l}\text { Frequentemente não-especificada, } \\
\text { potencialmente com viés }\end{array}$ & $\begin{array}{l}\text { Fontes abrangentes, estratégia de } \\
\text { busca específica }\end{array}$ \\
\hline Seleção & $\begin{array}{l}\text { Frequentemente não-especificada, } \\
\text { potencialmente com viés }\end{array}$ & $\begin{array}{l}\text { Seleção baseada em critérios } \\
\text { aplicados uniformemente }\end{array}$ \\
\hline Avaliação & Variável & Avaliação criteriosa e reprodutível \\
\hline Síntese & Qualitativa & Quantitativa* \\
\hline Inferências & $\begin{array}{l}\text { Às vezes baseadas em resultados } \\
\text { de pesquisa clínica }\end{array}$ & $\begin{array}{l}\text { Frequentemente baseadas em } \\
\text { resultados de pesquisa clínica }\end{array}$ \\
\hline *Uma síntese quantitativa que inclui um método estatístico é uma análise \\
\hline
\end{tabular}

Fonte: Cook, 1997 apud. Rother (2007).

Desse modo, a revisão narrativa ou tradicional, contempla uma abordagem mais ampla e "dificilmente parte de uma questão específica bem definida, não exigindo um protocolo rígido para sua confecção" (CASTRO, 2007, não paginado). Também na revisão narrativa ou tradicional, "a busca das fontes não é pré-determinada e específica, sendo frequentemente menos abrangente" (CASTRO, 2007, não paginado). Igualmente, em relação a escolha dos artigos, cabe acrescentar que "é arbitrária, provendo o autor de informações sujeitas a viés de seleção, com grande interferência da percepção subjetiva" (CASTRO, 2007, não paginado). Esta situação, em muitos casos, pode ocasionar deduções imprecisas e falácias, vindo a prejudicar os resultados da pesquisa realizada. Ressalta-se ainda que nesse tipo de revisão, busca-se de imediato a análise da literatura selecionada e sua interpretação, visando ao estado da arte, como fator predominante no estudo de um determinado tema. E, por intermédio do levantamento bibliográfico, responde-se à problemática proposta para a investigação teórica.

Quanto à revisão sistemática, a busca por fontes abrangentes, pautada em estratégias de buscas específicas, promove uma seleção baseada em critérios aplicados uniformemente, com avaliação criteriosa e reprodutível, baseada em evidências e na validade científica dos estudos selecionados. 
Para tanto, recorre-se ao uso da técnica de fichamento, para a coleta dos dados e das informações contidas nas fontes bibliográficas, com o propósito identificar na literatura pertinente, os fundamentos teóricos para a sustentação dos argumentos defendidos sobre o objeto de pesquisa.

Geralmente, a revisão sistemática é um estudo retrospectivo, podendo, entretanto, ocorrer sob a forma de revisões sistemáticas prospectivas. Em relação à avaliação da qualidade de uma revisão sistemática, ocorre por meio de uma escala validada com indagações que variam entre os resultados obtidos e a fidelidade dos dados, considerando a precisão do risco existente e a aplicabilidade prática na população que se está estudando. (Quadro 3). Nesse sentido, convém acrescentar a possibilidade de reprodução do estudo realizado e seus desfechos clínicos:

Quadro 3. Lista de avaliação de uma revisão sistemática.

\begin{tabular}{|c|}
\hline Lista de Avaliação \\
\hline Os resultados do estudo são válidos? \\
\hline A revisão sistemática responde a uma questão clínica específica? \\
\hline Os critérios utilizados para a selecção dos artigos foram apropriados? \\
\hline Estudos relevantes foram esquecidos? \\
\hline A validade dos estudos incluídos foi avaliada? \\
\hline A avaliação é reprodutível? \\
\hline Os estudos têm resultados semelhantes? \\
\hline Quais são os resultados? \\
\hline Qual o resultado geral da revisão sistemática?
\end{tabular}

Fonte: Oxman, Cook, Guyatt, [s.d.] apud. Pocinho,2008.

Na mesma perspectiva a Lista de Avaliação deve ser complementada pela Escala da Qualidade, também validada, visando identificar os métodos e as estratégias de pesquisa utilizadas nos estudos selecionadas para a revisão sistemática, como também os critérios de inclusão, validação e seleção das fontes bibliográficas, associados aos achados pertinentes e suas combinações aleatórias. Incluem-se nessa abrangência, as conclusões alcançadas e a 
qualidade da revisão. Ressalta-se também a importância da manutenção do tema em estudo, atualizado de modo a contemplar as inovações metodológicas da área (Quadro 4):

Quadro 4. Escala de qualidade da revisão.

Sistemática. Escala de Qualidade (Oxman, 1991; Oxman, 1994)

\begin{tabular}{|c|}
\hline Os métodos de busca foram descritos? \\
\hline A estratégia de busca foi abrangente? \\
\hline Os critérios de inclusão foram descritos? \\
\hline O viés de seleção foi evitado? \\
\hline Os critérios de validade foram descritos? \\
\hline Os critérios de validade foram apropriados? \\
\hline Os métodos para a metanálise foram descritos? \\
\hline Os achados foram combinados apropriadamente? \\
\hline As conclusões são adequadas para os resultados encontrados? \\
\hline Qual foi a qualidade geral da revisão sistemática?
\end{tabular}

Fonte: POCINHO, 2008.

Cabe destacar a necessidade de que o título da revisão sistemática seja registrado na Colaboração Cochrane no endereço: <http://www.cochrane.org/docs/pma.htm\#DOCS>, por intermédio de formulário próprio, disponível nessa plataforma. Também é exigida a elaboração do projeto de revisão sistemática que deve ser iniciado "depois do registo do título da revisão. Frequentemente o grupo de revisão recomenda a realização de um curso e envia um modelo de projeto ou outros materiais impressos sobre o grupo e a Colaboração" (PINTO, 2013, p.1039), possibilitando a sua divulgação e utilização em diversos tipos e níveis de estudo e áreas do saber.

\subsection{Pontos positivos e negativos da revisão sistemática}

A revisão sistemática é aceita como método de estudo que se pauta no rigor acadêmico e científico, especialmente quanto à presença da verificação das evidências e diretrizes a serem adotadas em uma pesquisa científica, por intermédio da validação dos resultados encontrados na literatura pertinente. Diante disso, são inúmeras as vantagens e/ou os pontos positivos dessa metodologia e, de acordo com Light (1984); Dickersi; Berlin (1992); 
Bossel et al. (1994); Cook et al. (1997); Mulrow (1989); Castro et al. (2002); Antman et al. (1992); Gelber; Goldhirsch (1991), Mulrow (1987); Oxman; Guyatt (1988); Chalmers; Altman (1995) e Chalmers; Enkin; Keirse (1989) citados por Riera; Abreu e Ciconelli (2006, não paginado), elas perpassam as seguintes assertivas:

a) utiliza um método científico que pode ser reproduzido na prática clínica; b) evita a duplicação desnecessária de esforços, uma vez que quando se completa a revisão ela não precisa ser repetida por outro grupo;

c) pode ser atualizada periodicamente, sem maiores esforços, por meio da busca de novos ensaios clínicos publicados, que, se forem de boa qualidade, serão incluídos na metanálise;

d) reduz controvérsias na literatura, uma vez que não resulta do número de estudos favoráveis a determinada intervenção, mas da soma de todos os casos estudados;

e) antecipa o resultado de grandes ensaios clínicos que ainda esperam para serem realizados, devido às dificuldades técnicas e/ou financeiras;

f) pode detectar tratamentos inadequados em estágios mais iniciais de seu uso, portanto, salvando um grande número de pacientes de efeitos adversos de tratamentos desnecessários;

g) aumenta a acurácia dos resultados, diminuindo o intervalo de confiança e aumentando o número da amostra;

h) direciona futuros estudos para áreas deficientes em evidências;

i) economiza recursos em pesquisa clínica e assistência médica;

j) auxilia decisões para políticas de saúde;

k) exige trabalho em equipe, cooperação, e "negociação" de opiniões, o que nem sempre é fácil, embora seja uma habilidade fundamental a ser desenvolvida pelos profissionais da área da saúde.

Adentrando no âmbito das principais funções de uma revisão sistemática, assim especificam Carvalho, Oliveira e Ribeiro (2011, p. 231): 
-reconhecer e dar crédito à criação intelectual de outros autores; -indicar a qualidade da produção de conhecimento prévia na área; -abrir um espaço para ampliar e acrescentar conhecimentos já estabelecidos, mas que podem receber novas contribuições de pesquisas; -através da revisão de literatura é possível apontar e avaliar o conhecimento produzido em pesquisas prévias, destacando conceitos, procedimentos, resultados, discussões e conclusões relevantes para seu trabalho.

Apesar da relevância dos estudos de revisão sistemática e suas contribuições na construção e ampliação do conhecimento, ainda existem algumas desvantagens e/ou pontos negativos que, muitas vezes dificultam e interferem em sua execução, dentre eles: o período de realização, podendo alcançar entre três meses e um ano de duração; a necessidade de dedicação e mesmo, o esforço intelectual para identificar, interpretar e comparar os resultados obtidos; a impossibilidade de o pesquisador realizá-la sozinho e ainda, a falta de qualidade dos estudos utilizados na revisão e em especial, os relativos a ensaios clínicos (ATALLAH, 1997; COOK; MULROW; HAYNES, 1997; NAYLOR, 1995 apud RIERA; ABREU; CICONELLI, 2006, não paginado).

Ressalta-se que para garantir a qualidade de uma revisão sistemática, "é necessário definir o conjunto de critérios que foram adotados no planejamento na execução e na análise do levantamento bibliográfico, a fim de minimizar vieses, onde é possível evidenciar que a eficácia/efetividade de uma intervenção pode ser sub ou superestimada quando a qualidade da revisão não for satisfatória" (CARVALHO; OLIVEIRA; RIBEIRO, 2011, p. 232).

Assim, elaborar uma revisão sistemática requer dedicação e rigor intelectual e científico, devendo-se considerar em sua confecção: “a) os verbos utilizados pelo autor nas citações; b) a relação entre as pesquisas citadas (se sobrepõem/ contrastam entre si); c) se justifica a presença dos textos citados; d) se explicita em que momento se é o único autor do texto que está sendo construído" (CARVALHO; OLIVEIRA; RIBEIRO, 2011, p. 234), entre outros aspectos. Além disso, faz-se necessária a existência de diálogo entre os autores citados com o 
pesquisador mediador e deles entre si, para que ocorra a revisão dos conteúdos abordados e dos respectivos resultados encontrados, com o intuito de garantir a sua validade.

\section{METANÁLISE}

No âmbito da pesquisa bibliográfica, o termo Metanálise representa "a análise estatística de uma coleção de resultados de estudos individuais, com o objetivo de integrar os resultados" (CASTRO, 2007, não paginado) encontrados na literatura pertinente. Etimologicamente falando, "[...] [o] prefixo meta tem origem no grego e significa "além", "transcendência", "reflexão crítica sobre". A grafia da palavra frequentemente gera discussão. O termo metaanalysis foi incluído entre os Medical Subject Headings (PubMed) ou Descritores em Ciência da Saúde (Biblioteca Virtual em Saúde) em 1992, o que permite a utilização desse descritor para identificar metanálises publicadas na Medline e na Lilacs (CASTRO, 2007, não paginado).

Nessa perspectiva, para que "o produto de uma metanálise, como síntese quantitativa de uma revisão, seja fidedigno, é extremamente importante que os estudos-fonte dos dados originais tenham sido avaliados" (CASTRO, 2007, não paginado), considerando nesse âmbito a utilização de "critérios tais que se possa confiar na adequação de sua associação com a finalidade de chegar a um resultado integrado" (CASTRO, 2007, não paginado). Com o intuito de garantir fidelidade ao objeto pesquisado, assim possibilitando a verificação das evidências e, consequentemente, a segurança em relação à solução da problemática investigada.

Portanto, pode-se afirmar que a metanálise, em sua essência é uma técnica estatística, utilizada em estudos quantitativos e também qualitativos, principalmente por possibilitar uma postura capaz de:

Combinar resultados provenientes de diferentes estudos produzindo, assim, estimativas que resumem o todo, chamadas de estimativas metanalíticas. Para que o resultado de uma metanálise tenha 
significado aplicado, os estudos que compõem os dados da metanálise devem ser o resultado de uma revisão sistemática (RODRIGUES, 2010, p.436).

Significando, em outras palavras que a metanálise, "é uma abordagem estatística que combina resultados de estudos relevantes para responder uma questão" (BERWANGER et al., 2007, p.476). Além disso, de forma mais abrangente, enquanto método que busca as evidências disponíveis, consistindo em sintetizar "e apontar áreas onde há necessidade de mais pesquisas" (BERWANGER et al., 2007, 477). Para tanto, faz uso de métodos estatísticos na análise dos dados alcançados, com o intuito de "sumarizar os resultados de diversos estudos em uma única medida denominada, genericamente, estimativa de efeito conjunto" (BERWANGER et al., 2007, 478).

São métodos utilizados nas revisões sistemáticas na área de saúde: Parâmetro clínicoepidemiológico de sumarização dos resultados e Método de quantificação da contribuição dos estudos para análise. Porém, em estudos

com maior poder estatístico (leia-se, estudos com maior população e maior efeito da intervenção) possuirão mais "peso". Nesse caso, utiliza-se o método de efeitos fixos, que pressupõe que todos os estudos apontaram um mesmo efeito. Quando há diversidade e heterogeneidade, é utilizado o modelo de efeitos-aleatórios, que distribui o peso de maneira mais uniforme, valorizando a contribuição dos estudos pequenos e, consequentemente, fornece um intervalo de confiança (IC) mais amplo (BERWANGER et al., 2007, 479).

Igualmente, cabe acrescentar que os resultados de metanálise, quase sempre são representados por um gráfico do tipo Forest Plot (diagrama), com a finalidade de concatenar "em uma única figura, todas as informações sobre o efeito/precisão do tratamento e a contribuição de cada estudo para a análise" (BERWANGER et al., 2007, 479). De modo geral, 
busca-se na leitura de metanálise a "estimativa sumária, resultante da compilação dos diferentes trabalhos" (CORREIA, 2015, não paginado) publicados, representada pelo diamante (losango) que aparece na parte inferior do gráfico, indicando o resultado final da combinação dos dados obtidos, visando determinar as evidencias presentes na heterogeneidade, representada pela variabilidade ou diferença entre eles e as estimativas de efeitos, por meio do uso de método estatístico.

Contemplando também as características dos participantes, intervenções e conclusões alcançados com a pesquisa realizada. Correia explica a presença da heterogeneidade nos estudos de metanálise da seguinte maneira:

A melhor forma de avaliar essa veracidade seria repetir o experimento várias vezes e ver se o mesmo resultado se repete. Mas isso não ocorre, quem faz um ensaio clínico só faz uma vez e publica. O que faz uma metaanálise é simular esta repetição de experimentos, utilizando os estudos que existem na literatura. Hipoteticamente, ao realizar uma meta-análise, estamos repetindo o experimento várias vezes. E nesta repetição, avaliamos heterogeneidade entre os estudos, vendo se o resultado se repete nos diferentes experimentos. Se os experimentos se confirmam entre si, ficamos mais certos de que aquele resultado não decorreu do acaso (CORREIA, 2015, não paginado).

Desse modo, é possível identificar o "percentual da variação do resultado entre os estudos que ultrapassam o efeito do acaso" (CORREIA, 2015, não paginado). São vantagens dos estudos de metanálise: o aumento do percentual estatístico do resultado homogêneo, a maior precisão baseada em evidências e a possibilidade de responder a perguntas que não foram solucionadas pelos estudos primários. São considerados estudos primários, os ensaios clínicos aleatórios, estudos de acurácia, estudos coortes entre outros. Os estudos secundários contemplam os estudos de custo-minimização, de custo-efetividade, de custoutilidade e de custo-benefício. 
A realização satisfatória da metanálise necessita de pelo menos "dois estudos que respondam a uma mesma pergunta, utilizem pelo menos um desfecho em comum e tenham desenhos de estudo semelhantes" (SOUZA, 2015, não paginado). Cabendo ainda avaliar internamente a "extensão em que o desenho e a condução do estudo preveniram vieses" e externamente, "a extensão em que os resultados de um estudo podem ser generalizados" (SOUZA, 2015, não paginado). Por fim, na verificação dos dados e das informações coletadas, ressalta-se que, "[...] [a] análise estatística [...] só pode ser executada se os estudos incluídos forem semelhantes, ou seja, se a amostra, a intervenção e os desfechos clínicos forem homogêneos. Se a combinação dos estudos não fizer sentido clínico e metodológico, a metanálise não deve ser executada" (PINTO, 2013, p.1040).

Portanto, exigindo a presença dos níveis de evidência, por meio dos quais é possível avaliar e validar criticamente as publicações científicas selecionadas, visando à tomada de decisão sobre uma determinada problemática. Situação em que as evidências são hierarquizadas e verificadas criticamente, de acordo com os diferentes enfoques, prognósticos e prevalências. Igualmente, constituindo um acervo literário de grande valia para o estudo de um questionamento que necessita ser solucionado, especialmente, quando oriundo de situações que geram incertezas e/ou inseguranças.

Quanto ao aspecto da sustentabilidade científica, a revisão sistemática recorre aos resultados obtidos com a metanálise, a partir da identificação e da interpretação das principais publicações acerca do tema na literatura científica. Igualmente, por intermédio da organização das ideias, visando à possibilidade de avanços na área de estudo, permeia a promoção prática de novas conexões literárias que ampliam as discussões e os questionamentos pautados nas evidências que embasam determinado tema. 


\subsection{Metanálise qualitativa}

Nas Ciências Humanas e Sociais, a metanálise contempla a utilização de categorias preestabelecidas para uma temática em estudo, visando identificar semelhanças e controvérsias em uma determinada quantidade de publicações. Por sua vez, a vertente qualitativa pauta-se na possibilidade de visualização do impacto dos estudos realizados na área, sobre a questão investigada, ampliando assim, o nível de evidências, suas constatações e aplicabilidades.

Lembrando que nessa perspectiva, as evidências estão direcionadas para a explicação e a interpretação de determinado fenômeno, a partir da reunião dos resultados alcançados de outros estudos, prevalecendo em sua metodologia, à integração interpretativa que, segundo Castro citado por Pinto (2013), deve contemplar a seguinte sequência: a) formulação da pergunta; b) localização e seleção dos estudos; c) avaliação crítica dos estudos; d) coleta de dados; e) análise e apresentação dos dados; f) interpretação dos dados; g) aprimoramento e atualização da metanálise. Consideram-se nessa abordagem os problemas que não foram solucionados nos estudos avaliados. Num segundo momento, procede-se o agrupamento dos dados obtidos, podendo-se ou não, fazer uso de técnicas estatísticas.

Nesse sentido, cabe salientar que nos estudos de campo, prevalece a Teoria Fundamentada (TF), elaborada em 1967 por Glaser e Strauss citados por Pinto (2013), que contempla a coleta de dados por meio da entrevista. Em sua abordagem, especifica que "[...] [o] pesquisador analisa os dados de modo a entender determinada situação e como e por que seus participantes agem de determinada maneira, como e por que determinado fenômeno ou situação se desdobra deste ou daquele jeito" (PINTO, 2013, p.1041). A TF trata da análise dos estudos primários com diferentes perspectivas para o mesmo tema. Quanto a sua metodologia procedimental, recai sobre a utilização do Método Indutivo. 


\section{CONSIDERAÇÕES FINAIS}

Desse modo, pode-se afirmar que nas Ciências da Saúde a revisão sistemática:

[...]combina os resultados de cada estudo para obter uma estimativa global do efeito do tratamento, possibilitando a análise das fontes de heterogeneidade. Se os doentes, intervenções e desfechos clínicos de cada estudo são semelhantes, provavelmente, os resultados podem ser combinados. (POCINHO, 2008, p.4-5)

Superando, assim, a expectativa em relação à presença da sensibilidade esperada, por meio da utilização de metanálise, com informações úteis sobre um conteúdo específico, contido em dois ou mais estudos, gerando, consequentemente uma estimativa de efeito positivo.

Já no âmbito das Ciências Humanas, a revisão sistemática promove o acesso às informações publicadas, permitindo o cruzamento entre os resultados obtidos e as conclusões alcançadas nos variados estudos originários de diversas fontes, visando à elaboração de uma síntese integradora e interpretativa, para a compreensão da problemática investigada e o alcance de sua elucidação.

Quanto ao investigador, é importante que possua o conhecimento básico sobre a metodologia padrão da revisão sistemática e do método estatístico, associados ao conhecimento da questão investigada "para encontrar, avaliar e interpretar todas as pesquisas relevantes disponíveis "[...] [na] área do conhecimento ou fenômeno de interesse, que representa o atual conhecimento sobre a intervenção ou fator de exposição no momento da realização da revisão sistemática [...]" (BUEHLER et al., [s.d.], p.3,5). Igualmente, o domínio da informática e de pelo menos um idioma estrangeiro, preferencialmente a língua inglesa.

Devendo ainda, conhecer e dominar a utilização de "ferramentas necessárias à condução da 
revisão, como os gerenciadores de referência, os programas que geram a metanálise, as peculiaridades de busca nas várias bases de dados, redação do protocolo e do manuscrito" (BUEHLER et al., [s.d.], p. 7), com as evidências encontradas na literatura pesquisada, na área de atuação.

Portanto, pode-se afirmar ser a revisão sistemática, um tipo de estudo metodologicamente rigoroso em suas etapas de realização, que promove a identificação de níveis de estimativa confiáveis, podendo ser associado ao uso de metanálise enquanto ferramenta de suporte que possibilita a concretização de sua assertiva. 


\section{REFERÊNCIAS}

BERWANGER, O. e outros. Como avaliar criticamente revisões sistemáticas e metanálises? Revista Brasileira de Terapia Intensiva 475, vol. 19, n. 4, out./dez, 2007. Disponível em: <amib.com.br/rbti/download/artigo_2010614135112.pdf>. Acesso em: 15 abr. 2015.

BUEHLER, A. M. e outros. Diretrizes metodológicas: elaboração de revisão sistemática e metanálise de estudos diagnósticos de acurácia. Brasília: Ministério da Saúde, [s.d.]. Disponível em: <http://200.214.130.94/rebrats/publicacoes/dbrs_Diagn_v_final.pdf>. Acesso em: 15 maio 2015.

CASTRO A. A. Curso de revisão sistemática e metanálise. Em: CORDEIRO A.M. Revisão sistemática: uma revisão narrativa, Rev. Col. Bras. Cir., vol. 34, n. 6, Rio de Janeiro, nov./dez., 2007. Disponível em: <http://www.scielo.br/scielo.php?script=sci_arttext\&pid=S0100$69912007000600012>$. Acesso em: 10 mar. 2015.

CARVALHO, Anderson Santos; OLIVEIRA, Fabrício Borges; RIBEIRO, Elisa Antônia Ribeiro. Aspectos relevantes na confecção de uma revisão sistemática e metanálise. Evidência, Araxá, vol. 7, n. 7, p. 229-236, 2011. Disponível em: <uniaraxa.edu.br/ojs/index.php/evidencia/ article/viewFile/199/185>. Acesso em: 13 out. 2015.

CORREIA, L.C. Medicina baseada em evidências. 2015. Disponível em: <http://medicinabaseadaemevidencias.blogspot.com.br/2014_03_01_archive.html>. Acesso em: 10 maio 2015.

DE-LA-TORRE-UGARTE-GUANILO, Mônica Cecilia; TAKAHASHI, Renata Ferreira; BERTOLOZZI, Maria Rita. Revisão sistemática: noções gerais. Rev. Esc. Enferm. USP, 45(5):1260-6, 2011. Disponível em: <scielo.br/pdf/reeusp/v45n5/v45n5a33.pdf>. Acesso em: 24 set. 2015.

GALVÃO, C. M.; SAWADA, N. O.; TREVISAN, M.A. Revisão sistemática: recurso que proporciona a incorporação das evidências na prática da enfermagem. Rev. Latino-am. Enfermagem, 12(3):549-56, maio/jun. 2004. Disponível em: <scielo.br/pdf/rlae/v12 sci_arttext\&pid=S0104-07072008000400018>. Acesso em: 15 maio 2015.

MENDES, K. D.S.; SILVEIRA, R. C. de C. P.; GALVÃO, C. M. Revisão integrativa: método de pesquisa para a incorporação de evidências na saúde e na enfermagem. Texto contexto enferm., vol. 17, n. 4, Florianópolis, Oct./Dec. 2008 Disponível em: <http://www.scielo.br/scielo.php?script=n3/v12n3a14.pdf >. Acesso em: 10 maio 2015.

MUNZLINGER, Elizabete; NARCIZO; Fabricio Batista; QUEIROZ, José Eustáquio Rangel de. Protocolo de Revisão Sistemática. UFCG, [s.d.]. Disponível em: <elizabete.com.br/rs/Tutorial _IHC_2012_files/ProtocoloRevisaoSistematica.pdf>. Acesso em: 10 set. 2015. 
Sistematização de revisões bibliográficas em pesquisas da área de IHC. 2012. Disponível em: <www.elizabete.com.br/site/Projetos_e_Estudos/Paginas/Doutorado_(atual) _files/CapituloTutorial-Munzlinger-Narcizo-Rangel.pdf>. Acesso em: 15 set.2015.

PINTO, Cândida Martins. Metanálise qualitativa como abordagem metodológica para pesquisas em letras. Atos de Pesquisa em Educação - PPGE/ME. vol. 8, n. 3, p. 1033-1048, set./dez. 2013. Disponível em: <http://dx.doi.org/10.7867/1809-0354.2013v8n3p10331048>. Acesso em: 20 abr. 2015.

POCINHO, Margarida. Lições de metanálise, 2008. Disponível em: $<$ docentes.ismt.pt/ m_pocinho/Licoes_de_revisao_sistematica_e_metanalise.pdf $>$. Acesso em: 10 maio 2015.

RIERA, Rachel; ABREU, Mirhelen Mendes de; CICONELLI, Rozana Mesquita. Revisões sistemáticas e metanálises na reumatologia. Rev. Bras. Reumatol., vol. 46, suppl. 1, São Paulo, Jun. 2006. Disponível em: <http://www.scielo.br/scielo.php?pid=S048250042006000700003\&script=sci_arttext>. Acesso em: 13 out. 2015.

RODRIGUES, C. L. Metanálise: um guia prático. Clinical \& Biomedical Research, vol. 30, n. 4, 2010. Disponível em: <http://seer.ufrgs.br/index.php/hcpa/article/view/16571/10735>. Acesso em: 25 abr. 2015.

ROTHER, Edna Terezinha. Revisão sistemática $X$ revisão narrativa. Editorial. Acta paul. Enferm., Vol. 20, n. 2, São Paulo, Abr./Jun. 2007. Disponível em: <scielo.br/scielo.php? pid=S0103-21002007000200001\&script=sci_arttext>. Acesso em: 20 set.2015.

SAMPAIO, R.F.; MANCINI, M.C. Estudos de revisão sistemática: um guia para síntese criteriosa da evidência científica. Rev. bras. Fisioter., vol. 11, n. 1, São Carlos, [n.p.], Jan./Feb. 2007. Disponível em: <http://www.scielo.br/pdf/rbfis/v11n1/12.pdf>. Acesso em: 20 abr. 2015.

SOUZA, M. C. de. Métodos de síntese e evidência: Revisão sistemática e metanálise. INCA, 2015. Disponível em: <http://bvsms.saude.gov.br/bvs/publicacoes/inca/mirian_metodo_ de_sintese_e_evidencia.pdf $>$. Acesso em: 10 maio 2015. 